\title{
Pervasive Patient Health Monitoring System
}

\author{
J.Geetha Ramani \\ Associate Professor,Department of Electronics and Communication Engineering, \\ SNS College of Technology, Coimbatore, Tamil Nadu, India \\ J.M. Dharani, C. Divya, R. FareenFirdousFathima, A. RajoonathulMizriya \\ UG Student, Department of Electronics and Communication Engineering, \\ SNS College of Technology, Coimbatore, Tamil Nadu, India
}

\begin{abstract}
The exponentially growing health care cost and increasing patient have promoted a serious need to revolutionize health care systems. The need to bring health care cost into a sustainable range is urgent issue that needs to be addressed. One possible way to address the challenges facing the health care industry is by caring for patient in their environments such as their residences. A pervasive patient health monitoring (PPHM) system infrastructure is developed to provide remote monitoring of patients. PPHM is placed on integrated cloud computing and internet of things technology. Continuous monitoring is performed when an intensive monitoring is needed for patients. In this case, sensors continuously collect vital data and send it to the personal server. The on-demand monitoring occurs when a request from any authorized person within the system such as patients, doctors or nurses is generated. Experimental analysis have shown that the proposed frame work is efficient with very high classification accuracy. The database on patient's health is maintained and the data can be retrieved whenever needed. An alert system is provided in case of emergency.
\end{abstract}

Keywords- BASN, Cloud technology, IoT, PPHM.

\section{INTRODUCTION}

The genesis of patient care systems occurred in the mid-1960's. One of the first and most successful systems was the Technicon Medical Information System (TMIS), begun in 1965 as a collaborative project between Lockheed and El Camino Hospital in Mountain View, California. Designed to simplify documentation through the use of standard order sets and care plans, TMIS defined the state of the art when it was developed. More than three decades later, versions of TMIS are still widely used, but the technology has moved on. The hierarchical, menu-driven arrangement of information in TMIS required users to page through many screens to enter or retrieve data and precluded aggregation of data across patients for statistical analysis. Today's users have a different view of what can be done with data, and they demand systems that support those uses.

The rest of the paper is organized as follows. Literature survey is explained in section II. Existing system is explained in section III. Proposed system is explained in section IV.

\section{LITERATURE SURVEY}

Body Area Sensor Network Mobile Healthcare System (BASN m-health) is the use of sensors and actuators to monitor and improve patients' physical and mental health conditions in a networked system. It encompasses the use of mobile telecommunication and multimedia technologies integrated within mobile and wireless health care delivery systems. It regularly monitors patient normal daily activities. The cloud-based body area sensor network mobile health care system helps in achieving i. Early detection of infections; ii. Continuous monitoring of health status of people; and iii. Enhanced mobile scheduling between patients and healthcare professionals[6].

Health monitoring is an essential application of mobile sensor systems. Recent advances in sensor techniques, wireless communication, power supply technologies and wearable sensor systems have enabled the creation of a new generation of constant health monitoring. The Body Area Sensor Network (BASNS) are considered to be an important means to relieve the pressure of insufficient medical resources in an aging era and are becoming a strategic direction of the m-health research.

BASN is defined as a wireless network, which is formed by sensors located on, and/or biosensors transplanted into the human body and a data collector (Sink) used for medical data collection in real time. BASN can gather medical data, perform classified learning, and analyse data in real time, thus realizing an early medical warning.

The BASN m-health systems are based on the internet of things (IOT) whereby the medical treatment emphasizes the object management. This change of the concept urges the mobile medical technology to adapt "things" in the m-health systems which include doctors, patients, medical devices, and sensors. m-health 
broadly encompasses the use of mobile telecommunication and multimedia technologies as they are integrated within increasingly mobile and wireless health care delivery systems. It has been found that with the advent of m-Health; the cost savings were especially prevalent in the chronic disease areas of congestive heart failure, pulmonary disease, diabetes, and skin ulcers. With around the clock monitoring and electronic data transition to care-givers, remote devices speed up the treatment of patients requiring medical intervention.

IOT is an emerging technology, which permits devices and people correlated in an organized manner. In the era of IOT, the body sensor network technology is a part of it and has been rapidly increasing in the field of medical scope. Some of the applications of IOT are real time monitoring, patient information management and healthcare management. The patients are adhered with tiny sensors so that the patient information can be remotely monitored by the doctor. The patient health monitoring system creates aremote interaction between the patient and doctor. The tiny sensors like temperature, Heartbeat, Blood pressure are arranged in a manner to the patients to form an efficient Body sensor network [9].

The urge for the patient's health care management system is it can eliminate two dominant obstructions. First obstruction is that the doctor has to be on the site of the hospital for a long time. Second obstruction is that the patients are remained admitted in the hospitals for small health problems and may feel uncomfortable to stay in the hospital. The implementation of this system more no of patients can be supervised and better services can be contributed. The wearable tiny sensors are easily integrated with the human body in the patient health monitoring system and so that it can sense the physical parameters of the patient's body.

The temperature sensor, BP sensor, Heartbeat sensor is low cost and are having predominant circumstances in the patient health care management system. The BP, heartbeat, the temperature sensor is familiar and frequently used because every patient's health will primarily depend on these parameters. In general, every doctor is confined to support only one patient in real time, but by this system one doctor can monitor real time details of many people.

A large data fusion method based on wireless sensor networks is designed. Based on the analysis of the structure and learning algorithm of RBF neural networks, a heterogeneous RBF neural network information fusion algorithm in wireless sensor networks is presented. The effectiveness of information fusion processing methods is tested by RBF information fusion algorithm. The proposed algorithm is applied to heterogeneous information fusion of cluster heads or sink nodes in wireless sensor networks. The information fusion is defined as the computer processing system which makes full use of multi-sensor information resources of different time and space. Under certain criteria, computer technology is used to automatically analyze the observations of a number of sensors obtained by time series. This information process is carried out after optimization and synthesis to complete the required decision-making and estimation tasks. Sensors are the basis of information fusion. Multi-source information is the processing object of information fusion. Coordination, optimization and integrated processing are the core of information fusion [7].

Heterogeneous information processing needs to be carried out in a timely manner in order to utilize the information to obtain the exact state of the observation target or the complete real-time evaluation results. As a simplification and Simulation of human brain, neural network has powerful parallel processing ability. The RBF network with local excitation function can overcome the defects of the inherent property brought by BP algorithm that it is easy to get into local minimum. And its convergence is also easier to guarantee than the BP network, so the optimal solution can be obtained. Based on the analysis of the structure and learning algorithm of RBF neural networks, a heterogeneous RBF neural network information fusion algorithm in wireless sensor networks is presented. When it is used in heterogeneous information fusion of cluster head or sink node in wireless sensor networks, wireless transmission only needs to transfer the fusion results. This reduces the amount of data traffic, network conflicts and congestion.

\section{EXISTING SYSTEM}

PPHM is based on integrated cloud computing and Internet of Things technologies, which provide Healthcare systems with inexpensive but flexible and scalable pervasive technologies that enable long-term remote patient health status monitoring.

\section{A. BASN- BASED MOBILE HEALTH CARE SYSTEM-}

The BASN m-Health systems are based on the Internet of Things concept, whereby the medical treatment emphasizes the object management. This concept urges the mobile medical technology to adapt. "Things" in the $\mathrm{m}$-Health systems include doctors, patients, medical devices, and sensors. The focus of this research work is on 
the development of a cloud-based m-Health system, consisting of a healthcare middleware in the BASN domain and a distributed data processing system. The system contains three tiers as follows 1. Wearable Body Area Sensor Network (WBASN) 2. Automated Intelligent Central Node (AICN) or Sink 3. Cloud-based Central Server (CICS).

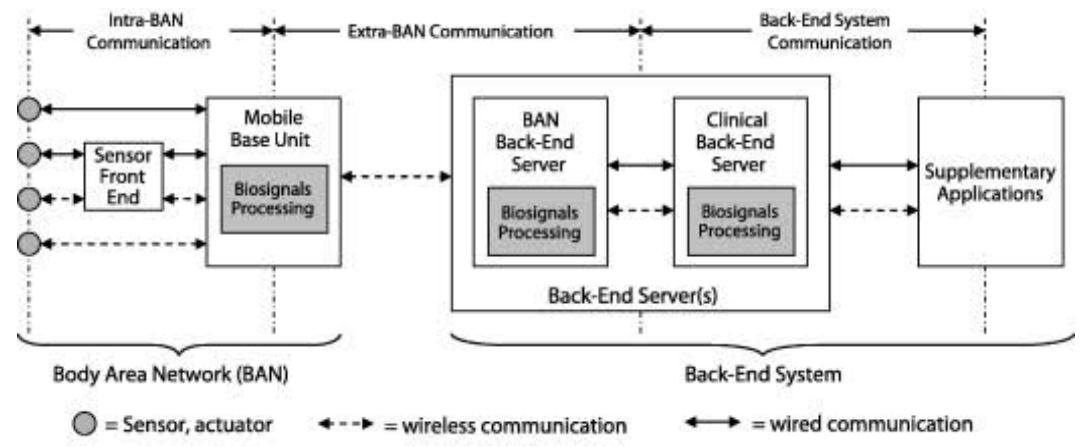

Figure 1.BASN framework

\section{B. REMOTE HEALTH STATUS MONITORING FRAME WORK-}

The observation station consists of an IOT subsystem that is tasked with remote physiological and activity monitoring of patients. These sensors can be implantable, worn or attached, to everyday objects such as clothes unobtrusively to gather specific physiological parameters such as a patient's blood sugar levels, blood glucose, capnography (i.e., $\mathrm{CO}_{2}$ level and breathing), and pulse oximetry and ECG continuously or on demand. Continuous monitoring is performed when intensive monitoring is needed for patients. In this case, sensors continuously collect vital data and send it to the personal server. The on-demand monitoring occurs when a request from any authorized person within the system, such as a patient, doctor, or nurse, is generated. The personal server provides a link between the IOT subsystem and the cloud infrastructure. The personal server is a dedicated per-patient machine (e.g., a tablet or smartphone) with built in features such as a GPS module, Bluetooth radio module, and SQLite database. We assume that the personal server can compatibly interact with various local networks such as Wi-Fi and LTE. Each sensor within a given BSN is wirelessly connected via a single hop to a dedicated personal server.

It performs basic data analysis and aggregation, generating alarm signals, making the data available to the entities subscribed to be notified (e.g., patient), or pushing the data (along with the location of the patient) out to the cloud for further analysis and sharing by healthcare professionals. In order to manage bandwidth and energy consumption, a fuzzy-based data fusion technique that distinguishes and aggregates only the true values of the sensed data is used.

This method decreases the processing and transmission of the sensed data as well as removes redundant data, thus minimizing energy depletion while prolonging the network lifetime. In addition to transferring data from the sensors to the cloud, the personal server can possibly receive a request for specific data from cloud applications or an end user.

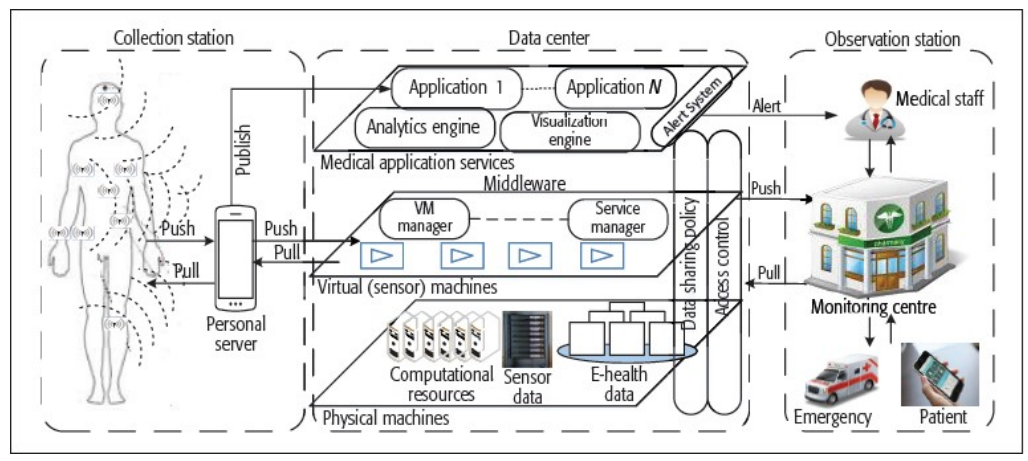

Figure 2. IOT \& Cloud-based architecture 
The observation station is where data-driven clinical observation and intervention take place. At this tier, entities such as healthcare professionals (e.g., doctors), emergency response services, medical research centres, and patients have the cloud also hosts the middleware system, virtual sensors, and application services that allow medical staff to analyse and visualize patients' data as well as to identify and raise alerts when events requiring urgent intervention are observed. The monitoring center involves the participation of many healthcare actors, including doctors, patients, and nursing staff, in clinical observation, patient diagnosis, and intervention processes. Thus, all access requests for patient data are managed by the monitoring center. Any authorized user wanting to access the sensor data can do so by issuing a data request to the cloud through the monitoring center. If the requested data is available in the sensor data storage, the data will be returned to the user.

\section{DATA CENTER SUBSYSTEM-}

The cloud relieves the IOT subsystem by performing heavy functions that require storing, processing, and analyzing the collected patient health data from the IOT subsystem. Cloud storage offers benefits of scalability and accessibility on demand at any time from any place. The healthcare provider data center hosts the cloud subsystem, which delivers storage resources and provides computational capability for analyzing and processing of the collected data. The cloud also hosts the middleware system, virtual sensors, and application services that allow medical staff to analyze and visualize patients' data as well as to identify and raise alerts when events requiring urgent intervention are observed.

\section{PATIENT DATA STORAGE-}

The cloud storage resources are used for long-term storage of patients' medical information (e-Health) and the data from the IOT subsystem (sensory data). E-Health contains the conventional clinical data (e.g., clinic observation and lab test results) while the sensory data contains longitudinal patient data provided by BSNs. Based on the access control configuration, healthcare practitioners or emergency centers can access the stored information without visiting the patient. The physicians, having access to the sensory data along with the eHealth data supported by decision support systems, can improve the quality of patient health in remote locations by making better and quicker prognoses, intervention, and treatment recommendations.

\section{E. HEALTH DATA SHARING POLICY (HDSP)-}

One of the aims of the healthcare service providers for collecting clinical data from patients is to share them with authorized healthcare professionals. As data security and privacy are important issues in healthcare systems, we use an access control mechanism (e.g., signature or certificate) that ensures only legitimate end users can access the data in the cloud. We also use policy to control the sharing of data. HDSP governs how the patient data is shared among the authorized entities and used to verify the identity of the user with access authority. For instance, the policy can define that access to the sensor reading in the sensor data storage and the corresponding analysis results can only be accessed by the doctors in the neurology department. HDSP also ensures that patient unique identities and associated profiles should be anonymized before the data is shared with other entities such as a research center. In the proposed framework, the data monitoring unit is responsible for setting up the HDSP taking into account regulatory compliance requirements and the need for sharing to provide the best possible care for the patient.

\section{F. CLOUD MIDDLE WARE-}

The middleware consists of a virtual machine (VM) manager and a service scheduler, among others. The VM manager is responsible for managing the virtual sensors, which are virtualized counterparts of physical sensors in BSNs, collecting sensor data from personal servers, and storing those data in the "sensor data" store. As compared to the standard cloud workloads such as non-real-time data for scientific computation and storage, the workload from the IOT subsystem is characterized by high inter-arrival rates and highly variant runtimes but with low parallelism.

\section{G. MEDICAL APPLICATION SERVICES-}

The cloud hosts various services that process clinical data collected from the IOT subsystem for clinical observation and intervention, and to dispatch ambulances or notify family members of patients. The analytics engine (AE) extracts features from the collected data and classifies the data to assist healthcare professionals to facilitate good patient care.

For the healthcare professionals to use the results from the AE to reach accurate and appropriate responses and actions, the output from $\mathrm{AE}$ will be used by the visualization engine to make the data accessible to the 
healthcare professionals in a readily digestible format. The alert system raises alert signals when events requiring urgent interventions are observed. The alarm function generates alerts if the value of the sensed physiological parameters exceeds a predetermined threshold value. For example, an alarm signal is generated when abnormalities such as arrhythmia or hypotension are detected. This capability enables patient health problems to be detected without visiting a doctor, notifying healthcare providers if a check-up is needed, and generating emergency alerts to ambulances.

\section{PROPOSED SYSTEM}

\section{A. INTRODUCTION-}

PPHM framework is proposed for continuous monitoring of patients health. The sensors collect the data and send it to the personal server. Data is stored in the data center and retrieved whenever needed.

\section{B. CLOUD TECHNOLOGIES-}

In proposed system, the patient health is monitored continuously and the database is maintained. The objective is to integrate IOT and cloud technologies and to provide a data monitored continuously to both data center and observation server simultaneously and to provide an alert system and data retrieval capability whenever needed.In this system, PIC microcontroller is used to control the operation of the system. Temperature sensor, pressure sensor, bio-potential electrode sensor are used to measure the health status.LCD display is used to display the measurements of sensors.Proteus software is used to analyse and display the readings taken.

Java Script is used to create a webpage for storing and displaying the database using IoT. PPHM framework is proposed for patient monitoring which integrates IoT and cloud technology.

PPHM framework is three-tiered with push-pull communication between the three tiers. Thus, in our model, an authorized healthcare professional can request and obtain the real time data collected by a particular sensor in an IoT subsystem.

\section{SOFTWARE-}

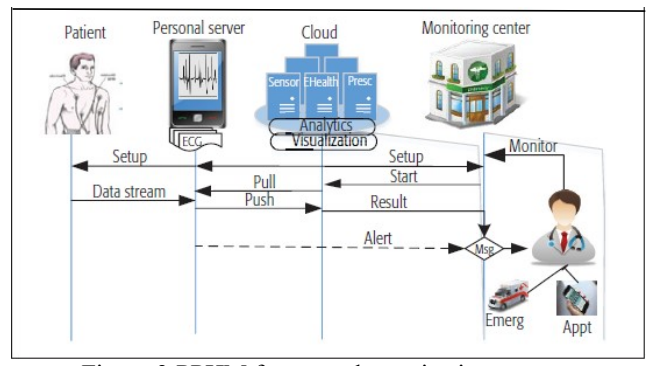

Figure 3.PPHM framework monitoring process

Proteus PIC Bundle is the complete solution for developing, testing and virtually prototyping the embedded system designs. A demonstration on the use of PROTEUSwill be given to you on this lab session, after that; you are encouraged to learn to use the software interactively.

The Proteus Design Suite is a Windows application for schematic capture, simulation, and PCB layout design. It can be purchased in many configurations, depending on the size of designs being produced and the requirements for microcontroller simulation. All PCB Design products include an auto router and basic mixed mode SPICE simulation capabilities.

D. $P I C-$

Peripheral interface controller also known as PICs, are used in electrical circuits and circuit that require a set amount of processing. They are inexpensive, simple to use, and the majority kind are flash so that they can be reprogrammed.

The PIC16F877A CMOS FLASH-based 8-bit microcontroller is upward compatible with PIC16C5x, PIC12Cxxx and PIC16C7x devices. It features 200ns instruction execution, 256 bytes of EEPROM data memory, self- programming, 2 comparators, 8 channels of 10-bit Analog-Digital convertor, 2 PWM functions, a synchronous serial port, a USART, and a parallel slave port.

\section{E. PRESSURE SENSOR-}


A pressure sensor measures pressure, typically of gases or liquids. Pressure is an expression of the force required to stop a fluid from expanding, and is usually stated in terms of force per unit area. A pressure sensor usually acts as a transducer; it generates a signal as a function of the pressure imposed. For the purposes of this article, such a signal is electrical.

Pressure sensors are used for control and monitoring in thousands of everyday applications. Pressure sensors can also be used to indirectly measure other variables such as fluid/gas flow, speed, water level, and altitude. Pressure sensors can alternatively be called pressure transducers, pressure transmitters, pressure senders, pressure indicators.

Pressure sensors can vary drastically in technology, design, performance, application suitability and cost. A conservative estimate would be that there may be over 50 technologies and at least 300 companies making pressure sensors worldwide.

\section{F. TEMPERATURE SENSOR-}

The LM35 series are precision integrated-circuit temperature sensors, whose output voltage is linearly proportional to the Celsius (Centigrade) temperature. The LM35 thus has an advantage over linear temperature sensors calibrated in ${ }^{\circ}$ Kelvin, as the user is not required to subtract a large constant voltage from its output to obtain convenient Centigrade scaling. The LM35 does not require any external calibration or trimming to provide typical accuracies of $\pm 1 / 4^{\circ} \mathrm{C}$ at room temperature and $\pm 3 / 4^{\circ} \mathrm{C}$ over a full -55 to $+150^{\circ} \mathrm{C}$ temperature range. Low cost is assured by trimming and calibration at the wafer level.

\section{G. ECG SENSOR-}

The electrocardiogram (ECG or EKG) is a diagnostic tool that is routinely used to assess the electrical and muscular functions of the heart. The electrocardiogram (ECG) has grown to be one of the most commonly used medical tests in modern medicine. Its utility in the diagnosis of a myriad of cardiac pathologies ranging from myocardial ischemia and infarction to syncope and palpitations has been invaluable to clinicians for decades.

Nervous stimuli and muscle contractions can be detected by measuring the ionic current flow in the body. This is accomplished using a bio-potential electrode.A negatively charged ion is an anion and a positively charged ion is a cation. The current flow in the human body is due to ion flow, not electrons. A bio-potential electrode is a transducer that senses ion distribution on the surface of tissue, and converts the ion current to electron current.

\section{H. LCD DISPLAY-}

There are many display devices used by the hobbyists. LCD displays are one of the most sophisticated display devices used by them. Once you learn how to interface it, it will be the easiest and very reliable output device used by you! More, for micro controller based project, not every time any debugger can be used. So LCD displays can be used to test the outputs.

Most of the LCD Displays available in the market are 16X2 (That means, the LCD displays are capable of displaying 2 lines each having 16 Characters a), 20X4 LCD Displays (4 lines, 20 characters). It has 14 pins. It uses 8 lines for parallel data plus 3 control signals, 2 connections to power, one more for contrast adjustment and two connections for LED back light.

\section{I. $\quad G S M-$}

GSM (Global System for Mobile Communications, originally Groupe Spécial Mobile), is a standard set developed by the European Telecommunications Standards Institute (ETSI) to describe protocols for second generation $(2 \mathrm{G})$ digital cellular networks used by mobile phones. It became the factor global standard for mobile communications with over $80 \%$ market share.

The GSM standard was developed as a replacement for first generation analog cellular networks, and originally described a digital, circuit switched network optimized for full duplex voice telephony. This was expanded over time to include data communications, first by circuit switched transport, then packet data transport via GPRS (General Packet Radio Services) and EDGE (Enhanced Data rates for GSM Evolution or EGPRS). Further improvements were made when the 3GPP developed third generation (3G) UMTS standards followed by fourth generation (4G)LTE Advanced standards.

\section{J. POWER SUPPLY-}

A power supply (sometimes known as a power supply unit or PSU) is a device or system that supplies electrical or other types of energy to an output load or group of loads. The term is most commonly applied to 
electrical energy supplies, less often to mechanical ones, and rarely to others. The transformer steps up or steps down the input line voltage and isolates the power supply from the power line.

\section{RESULT AND DISCUSSION}

The patient health is monitored continuously using sensors. The data is sensed and stored in cloud for future retrieval. The data is monitored continuously and immediate alert is sent to the doctor in case of emergency.

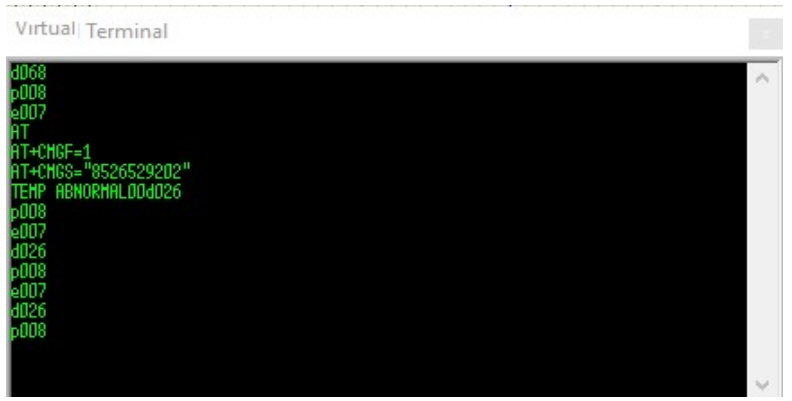

Figure 4.Simulation output-Patient monitoring

\section{CONCLUSION}

The system that integrates the capabilities of the IoT and cloud technologies for remote monitoring of patient's health status is proposed for monitoring patient health. The healthcare spending challenges by substantially reducing inefficiency and waste as well as enabling patients to stay in their own homes and get the same or better care is achieved.

\section{REFERENCES}

[1] Abawajy, J.H., Kelarev, A.V. and Chowdhury, M., (2013). Multistage approach for clustering and classification of ECG data. Computer methods and programs in biomedicine, 112(3), pp.720-730.

[2] Chowdhury, M., Abawajy, J., Kelarev, A. and Jelinek, H., (2016). A clustering-based multi-layer distributed ensemble for neurological diagnostics in cloud services. IEEE Transactions on Cloud Computing.

[3] Fortino, G., Parisi, D., Pirrone, V. and Di Fatta, G., (2014). BodyCloud: A SaaS approach for community body sensor networks. Future Generation Computer Systems, 35, pp.62-79.

[4] Ghanavati, S., Abawajy, J. and Izadi, D., (2016), July. An alternative sensor Cloud architecture for vital signs monitoring. In Neural Networks (IJCNN), 2016 International Joint Conference on (pp. 2827-2833). IEEE.

[5] Hossain, M.S. and Muhammad, G., (2016). Cloud-assisted industrial internet of things (iiot)-enabled framework for health monitoring. Computer Networks, 101, pp.192-202.

[6] Hassan, M.M., Lin, K., Yue, X. and Wan, J., (2017). A multimedia healthcare data sharing approach through cloud-based body area network. Future Generation Computer Systems, 66, pp.48-58.

[7] Izadi, D., Abawajy, J.H., Ghanavati, S.andHerawan, T., (2015). A data fusion method in wireless sensor networks. Sensors, 15(2), pp.2964-2979.

[8] Kaur, P.D. and Chana, I., (2014). Cloud based intelligent system for delivering health care as a service. Computer methods and programs in biomedicine, 113(1), pp.346-359.

[9] Luo, S. and Ren, B., (2016). The monitoring and managing application of cloud computing based on Internet of Things. Computer methods and programs in biomedicine, 130, pp.154-161.

[10] Ray, B.R., Chowdhury, M.U. and Abawajy, J.H., (2016). Secure object tracking protocol for the Internet of Things. IEEE Internet of Things Journal, 3(4), pp.544-553

[11] R. ArunSekar, G. Naveen Balaji, A. Gautami, B. Sivasankari "High Efficient Carry Skip Adder in various Multiplier Structures" Advances in Natural and Applied Sciences (Annexure II), Vol. 10 Issue 14 (Special) (Oct 2016) pp: 193-197, ISSN: 1995-0772

[12] M. Srinivasaperumal, K. Boopathi Raja, G. Naveen Balaji, E. Christina Dally "Concurrent Node Recovery From Failure In Wireless Sensor-Actor Networks” KARI Research Journal, Vol. 1 Issue 4 (Oct - Dec 2016) pp: 28-33, ISSN: $2456-6136$

[13] Naveen Balaji, R. Prabha, E. Shanthini, J. Jayageetha, Mohand Lagha "Rapid low power Synchronous circuits using transmission gates" Advances in Natural and Applied Sciences (Annexure II), Vol. 10, Issue 17 (Dec 2016) pp: 287-291, ISSN: 1995-0772

[14] G. Naveen Balaji, S. Chenthur Pandian, D. Rajesh "Fast Test Pattern Generator using ATALANTA M 2.0" Asian Journal of Research in Social Sciences and Humanities (Annexure I) Vol. 7 No. 2 (Feb 2017) pp. 721-729 ISSN: 2249-7315 10.5958/2249-7315.2017.00124.1

[15] G. Naveen Balaji, V. Aathira, K. Ambhikavathi, S. Geethiga, R. Havin "Low Power and High Speed Synchronous Circuits using Transmission Gates" Asian Journal of Research in Social Sciences and Humanities (Annexure I), Vol. 7 No. 2 (Feb 2017 ) pp. 713-720. ISSN: 2249-7315, DOI: 10.5958/2249-7315.2017.00123.X

[16] G. Naveen Balaji, S. Chenthur Pandian, D. Rajesh "A survey on effective Automatic Test Pattern Generator for self-checking Scan BIST VLSI circuits" International Research Journal of Engineering and Technology,Vol. 3, Issue 5 (May 2016) pp: 645-648, ISSN: $2395-0056$ 